\title{
Détermination de la conductivité hydraulique d'un limon non saturé
}

\section{Determination of the hydraulic conductivity of an unsaturated silt}

\author{
S. ED DINY, F. MASROURI, J.-P. TISOT* \\ GRECO Géomatériaux
}

Rev. Franç. Géotech., n 62, pp. $67-74$ (janvier 1993)

\section{Résumé}

Deux méthodes de mesure de la conductivité hydraulique sont exposées. La première, basée sur le suivi du débit sortant lors de l'application d'une pression d'air (succion), permet d'étudier le couplage entre les variations volumétriques de l'échantillon et celles du volume d'eau sortant. Afin de mesurer très précisément ces petites variations, qui, bien évidemment, nécessitent un long temps d'expérience, un système de mesure de haute précision a été utilisé. La seconde méthode repose sur le principe des profils instantanés et permet de couvrir une large gamme de succion, les mesures sont effectuées en drainage et en humidification par la méthode du papier filtre.

\section{Abstract}

The results of two methods of hydraulic conductivity measurements are presented. The first one is based on the study of the outflow caused by air pressure (suction), during this test, the both-relation between volumetric change of sample and the volumetric variation of water is shown in relief. In ordre to measure accurately these volume changes during a long time, a high precision system is used. The second method is based on the principle of instantaneous profils and allows to study a large scale of suction, measurements are realized during drying and wetting.

\footnotetext{
- Laboratoire de Géomécanique, BP 40, ENSG, 54501 Vandœuvre-les-Nancy.
} 


\section{INTRODUCTION}

Des phénomènes très complexes dus à la nature multiphasique des sols non saturés régissent la dynamique des écoulements dans ce type de matériau. En effet, entre la surface du sol et la nappe phréatique existe une zone qui contrôle tous les transferts mais pour laquelle les mécanismes d'écoulement sont encore mal connus ; c'est la zone aérée ou non saturée, caractérisée par l'existence de deux phases non miscibles dans la matrice poreuse du sol: l'air et l'eau. En fonction des écoulements, les proportions volumiques entre ces deux phases varient continuellement. La présence d'une interface entre la phase liquide (eau) et la phase gazeuse (air) crée des effets de pression capillaire qui doivent être pris en compte dans les équations de transferts.

Cependant, la pression capillaire dépend (par la courbure des interfaces) du volume du liquide contenu dans un pore. Le coefficient de transfert du liquide (conductivité hydraulique) est lui-même fonction de la dimension des chenaux liquides (dimension des pores), donc de la concentration volumique en liquide.

Ainsi le comportement des matériaux et des ouvrages étant entièrement lié à l'évolution de la teneur en eau des terrains, il importe de prévoir les échanges d'humidité à l'intérieur de ces corps. Des problèmes identiques se posent lors de la mise en ouvre des terres (remblais, talus, barrage), la stabilité des structures dépendant, dans l'immédiat des états initiaux des matériaux et à long terme de l'évolution des propriétés de ceux-ci. Le mécanisme des échanges d'humidité des sols dans ces conditions fait intervenir à la fois les forces de rétention de l'eau dans les sols (les forces de succion) et la perméabilité ou conductivité à l'eau.

Les expériences de détermination directe de la conductivité en milieu non saturé sont encore assez peu nombreuses. Souvent cette conductivité est déduite du bilan de volume effectué au cours du drainage et en utilisant la technique des profils instantanés. WESSELING et al. (1966), VACHAUD (1968), VAUCLIN (1975) et DANIEL (1983), en utilisant des psychomètres, des tensiomètres ou des rayons gamma ont réalisé des essais basés sur cette méthode. D'autres chercheurs ont essayé de mesurer la conductivité hydraulique en régime permanent (KLUTE, 1965), mais la méthode la plus utilisée pour déterminer la conductivité au laboratoire consiste à mesurer le débit sortant d'un échantillon placé dans une chambre à pression d'air contrôlé (GARDNER, 1956; MILIER et ELRICK, 1958; KUNZE et KIRKHAM, 1962 ; PECK, 1966).

Le but de ce travail est de caractériser les propriétés hydrauliques d'un sol fin non saturé: le limon de Jossigny. Les deux méthodes de mesure en régime transitoire décrites ci-dessus seront présentées.

\section{SOL ÉTUDIÉ}

Le matériau utilisé est le limon de Jossigny $(30 \mathrm{~km}$ à l'Est de Paris): $\gamma_{\mathrm{s}}=27,3 \mathrm{kN} / \mathrm{m}^{3} ; \mathrm{w}_{\mathrm{L}}=37,3 \%$; $\mathrm{w}_{\mathrm{p}}=28 \% ; \gamma \max =17 \mathrm{kN} / \mathrm{m}^{3} ; \mathrm{w}_{\text {opt }}=16,5 \%$

\section{MÉTHODE DES PROFILS INSTANTANÉS}

\subsection{Principe}

Cette méthode consiste à mesurer en fonction du temps les profils de succion et/ou d'humidité, ce qui permet d'évaluer les flux à différents intervalles de temps et d'espace par intégration graphique entre les profils successifs d'humidité (ou de succion). La détermination de la conductivité hydraulique nécessite la connaissance de:

- lévolution des teneurs en eau (ou des succions) pour différentes sections en fonction du temps;

- la vitesse d'avancement du profil d'humidité (ou de succion), cette vitesse est directement donnée par la pente de la courbe $x=f(\theta, t)$ où $x$ est la distance par rapport à la base de l'échantillon, $\theta$ est la teneur en eau volumique et $t$ le temps ;

- le gradient de succion: par inversion de paramètre et en utilisant la courbe de rétention de l'eau du sol, on peut obtenir la répartition du potentiel au cours du temps.

\subsection{Etat initial des échantillons}

Les deux échantillons testés présentent les propriétés initiales suivantes:

- essai $n^{\circ} 1$ (compactage dynamique): $w=21 \%$; $\gamma_{d}=16,4 \mathrm{kN} / \mathrm{m}^{3} ; e=0,664 ; \mathrm{k}_{\text {sat }}=10^{-9} \mathrm{~m} / \mathrm{s}$

- essai $n^{\circ} 2$ (compactage statique) $: w=28 \%$;

$\gamma_{d}=15,64 \mathrm{kN} / \mathrm{m}^{3} ; e=0,746$;

$\mathrm{k}_{\mathrm{sat}}=2.10^{-9} \mathrm{~m} / \mathrm{s}$

\subsection{Dispositif expérimental (fig. 1)}

Il est composé d'un cylindre (hauteur $21 \mathrm{~cm}$; diamètre $8 \mathrm{~cm}$ ) découpé en tranches de $3 \mathrm{~cm}$ d'épaisseur. Chaque élément de la colonne est carotté directement

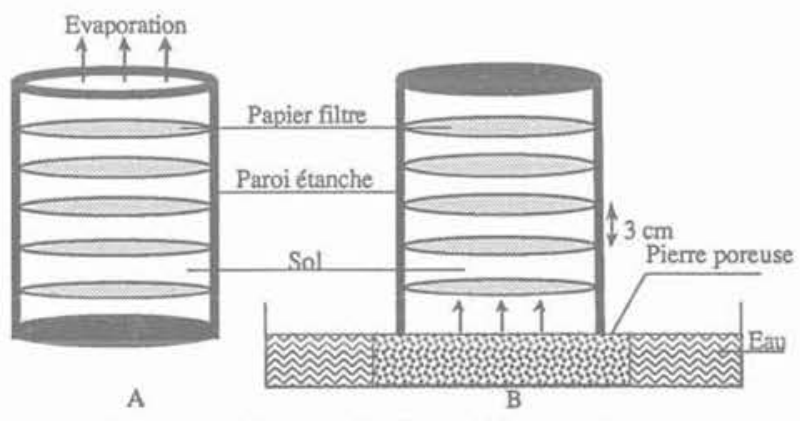

Fig. 1. - Schéma du dispositif pour la mesure de la conductivité hydraulique.

$A$ : en drainage : $B$ : en humidification.

Fig. 1, - Fitting for measurement of hydraulic conductivity.

A: drying: B: wetting. 
dans le moule qui sert à un compactage statique ou dynamique du limon. Entre deux tranches successives trois papiers filtres ont été placés; les mesures sont effectuées uniquement sur le papier du milieu qui reste protégé par les deux autres. La succion à chaque niveau est mesurée par la méthode du papier filtre (SIBLEY et al. 1990)

Une fois la colonne du sol fabriquée et les papiers filtres mis en place, on a fermé le sommet du cylindre; cette procédure permet d'obtenir, après 15 à 20 jours, une répartition homogène des teneurs en eau dans le profil du sol, c'est-à-dire d'atteindre un équilibre entre les papiers filtres, qui sont saturés au départ, et les différentes parties de la colonne du sol.

\subsection{Evaporation}

\subsubsection{Description des essais}

Après l'équilibre (homogénéisation), on permet l'évaporation par le sommet du cylindre (fig. 1A) et en fonction du temps on note le poids des papiers filtres des différentes profondeurs ainsi que le poids total de la colonne. A la fin de l'essai, on détermine le poids sec des papiers ce qui permet de déterminer leurs teneurs en eau et d'en déduire les succions en se référant à la courbe d'étalonnage du papier filtre.

En utilisant la courbe de rétention de l'eau du sol, on peut convertir les succions, mesurées précédemment, en teneurs en eau et par conséquent connaître les variations au cours du temps ce qui donne directement la vitesse d'évaporation. La conductivité hydraulique est déduite en utilisant la loi de Darcy pour un écoulement non saturé, vertical et monodimensionnel :

$$
\mathrm{V}=-\mathrm{k}_{\mathrm{w}(\mathrm{s})} \frac{\mathrm{d} \varphi}{\mathrm{d} z}
$$

où $\mathrm{V}$ est la vitesse d'évaporation, $\mathrm{k}_{\mathrm{w}}$ la conductivité hydraulique, $\mathrm{z}$ la profondeur, $\mathrm{s}$ la succion et $\frac{\mathrm{d} \varphi}{\mathrm{d} z}$ le gradient.

Dans cette expression $\varphi$ regroupe l'effet de la pesanteur et de la succion, et puisque ces deux paramètres ont des effets opposés l'équation (1) devient alors:

$$
-\mathrm{k}_{w(\mathrm{~s})} \frac{\mathrm{d}}{\mathrm{dz}}(\mathrm{S}-\mathrm{z})=-\mathrm{k}_{w(\mathrm{~s})}\left(\frac{\mathrm{ds}}{\mathrm{dz}}-1\right)
$$

\subsubsection{Résultats}

Lorsque le sommet du cylindre commence à sécher il y a création d'un gradient de succion, ceci est montré sur la figure 2 où lon observe que le gradient total augmente avec le temps. On note que le profil T1 est vertical, c'est-à-dire qu'au début de l'essai il y a la même succion aux différentes profondeurs ce qui montre que l'équilibre hydrique existe avant le début de l'évapora. tion. Après, l'évaporation diminue quand le sol se dessèche et donc il $y$ a une petite augmentation du gradient et une diminution de la vitesse d'avancement des profils, on note qu'entre le profil T4 et le profil T5 il y a un écart de 18 jours alors que cet écart n'est que de 2 à 3 jours entre les premiers profils. Cette méthode donne des profils de succion approximativement naturels, il n'y a pas une grande différence entre les drainages in situ et au laboratoire.

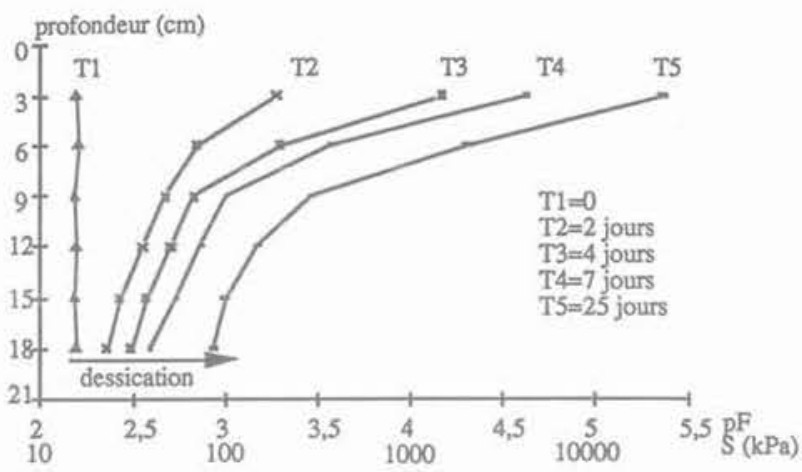

Fig. 2. - Les profils de succion (Essai no 1). Fig. 2. - Suction profils (Test no 1).

Le gradient de succion est donné par la pente de la courbe profondeur-log s (s est la succion en $\mathrm{cm}$ d'eau) en utilisant la formule suivante:

$$
\frac{\mathrm{ds}}{\mathrm{dz}}=\frac{\mathrm{d} \operatorname{Ln} s}{\mathrm{~d} \log s} \frac{\mathrm{ds}}{\mathrm{d} \operatorname{Ln} s} \frac{\mathrm{d} \log s}{\mathrm{dz}}=2,34 \frac{\mathrm{d} \log s}{\mathrm{dz}} \mathrm{s}
$$

Dans le cas du profil de succion à l'instant T4 (fig. 2), par exemple, les gradients de succion sont de l'ordre de 52 à $15 \mathrm{~cm}$ de profondeur pour dépasser à 30000 à $3 \mathrm{~cm}$.

La figure 3 montre la variation de la conductivité hydraulique en fonction de la succion exprimée en pF (pF $=\log$ de la succion s en cm d'eau). Dans la partie des faibles valeurs de succion la conductivité hydraulique est pratiquement constante et égale à celle mesurée à l'état saturé, ceci est valable en dessous d'une valeur particulière de succion qui correspond approximativement à la valeur d'entrée d'air déterminée à partir de la courbe de rétention d'eau $(\mathrm{pF} \approx 2,5$ pour l'essai $1 ; \mathrm{pF} \approx 2$ pour l'essai 2). Une fois cette valeur dépassée, la conductivité hydraulique chute de plusieurs ordres de grandeur pour une faible variation de succion, cette diminution peut être expliquée par le fait qu'en dessus de la valeur d'entrée d'air la continuité de la phase gazeuse (air) commence à s'établir.

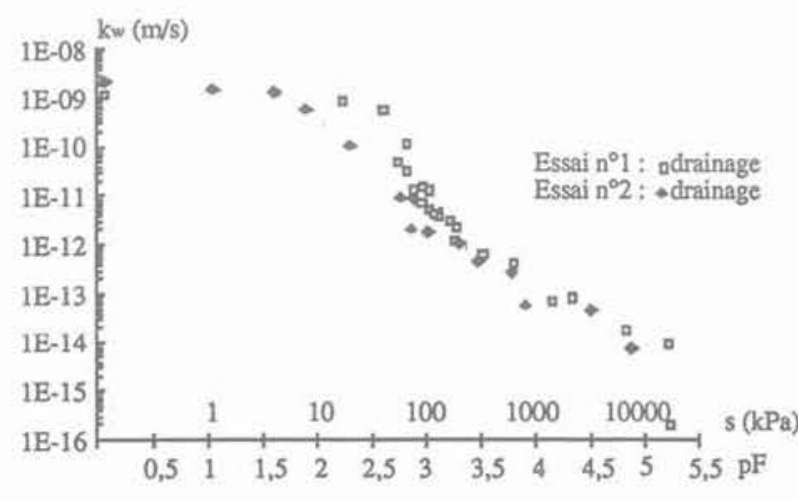

Fig. 3. - Evolution de la conductivité hydraulique en fonction de la succion.

Fig. 3. - Hydraulic conductivity versus suction. 
On constate que pour un sol dense (essai 1) la conductivité reste égale à celle mesurée à l'état saturé dans un domaine plus grand que celui du sol moins dense (essai 2), ceci serait dû au fait qu'au fur et à mesure que la succion se développe ce sont les pores les plus grands et donc les plus conducteurs qui se vident les premiers et l'écoulement de l'eau résiduelle ne se fait que dans les pores les plus petits. Ainsi, à l'état saturé, on sait qu'un sol lâche conduit l'eau plus rapidement qu'un sol dense, le contraire peut cependant être vrai lorsque les sols ne sont pas saturés. Pour les grandes valeurs de succion les conductivités se rappochent davantage.

Une étude parallèle de l'indice des vides, déterminé sur les échantillons servant à la mesure de la succion, a permis de tracer la fonction $k_{w}=f(e)$ (fig. 4, essai $n^{\circ} 2$ ). La courbe $\mathrm{k}_{\mathrm{w}}=\mathrm{f}(\mathrm{e})$ à l'état saturé, déterminée à l'œedo. mètre, est aussi rapportée sur la même figure. On remarque que, lors du drainage, la conductivité chute de plusieurs ordres de grandeur (10000 fois inférieur) pour une faible variation de l'indice des vides (de 0,7 à 0,55 ), alors qu'en condition saturée $\mathrm{k}$ ne varie que de deux ordres de grandeur (100 fois inférieur) pour des indices des vides variant de 0,5 à 0,87 . Cette observation nous permet de confirmer, qu'en condition non saturée, la conductivité est surtout influencée par le degré de saturation (FRY, 1992).

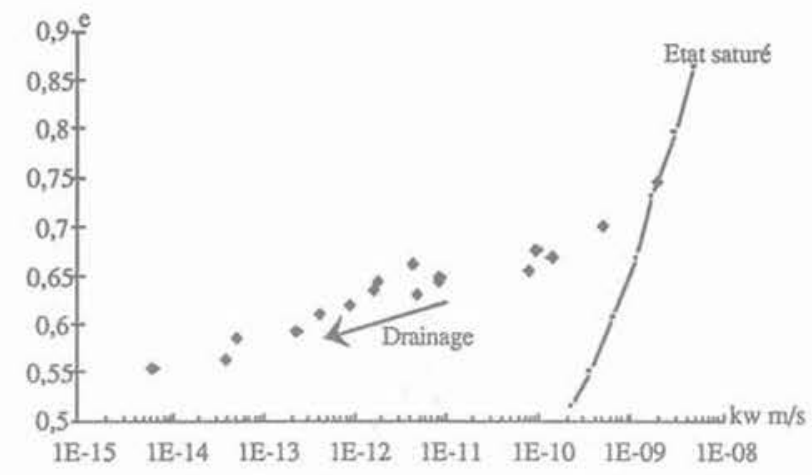

Fig. 4. - Variation de la conductivité saturée et non saturée en fonction de e.

Fig. 4. - Saturated and unsaturated hydraulic conductivity versus void ratio $e$.

\subsection{Humidification}

\subsubsection{Description des essais}

Le schéma du dispositif expérimental est représenté sur la figure $1 \mathrm{~B}$. Le cylindre est posé sur une pierre poreuse placée dans un bac d'eau régulièrement alimenté. La procédure expérimentale est identique au cas de l'évaporation à l'exception de la conversion des succions en teneur en eau qui s'effectue en se référant à la courbe de rétention d'eau en humidification. Ce qui nécessite l'établissement d'une courbe complète drainage-humidification du sol.

Une fois le drainage de la colonne terminé, on effectue immédiatement l'humidification à partir de la base, ceci en fermant le sommet du cylindre, la remontée de l'eau dans la colonne du sol se fait uniquement sous l'effet du gradient de succion.

\subsubsection{Résultats}

La figure 5 présente un cycle complet de drainagehumidification pour l'essai $n^{\circ} 1$, où l'on constate visiblement le phénomène d'hystérésis qui est classiquement attribué à plusieurs facteurs :

- la non uniformité géométrique des pores individuels ;

- l'effet de l'angle de contact, et le rayon de courbure qui est plus grand pour un ménisque qui avance;

- la présence d'air piégé qui tend à réduire elle aussi la teneur en eau des sols.

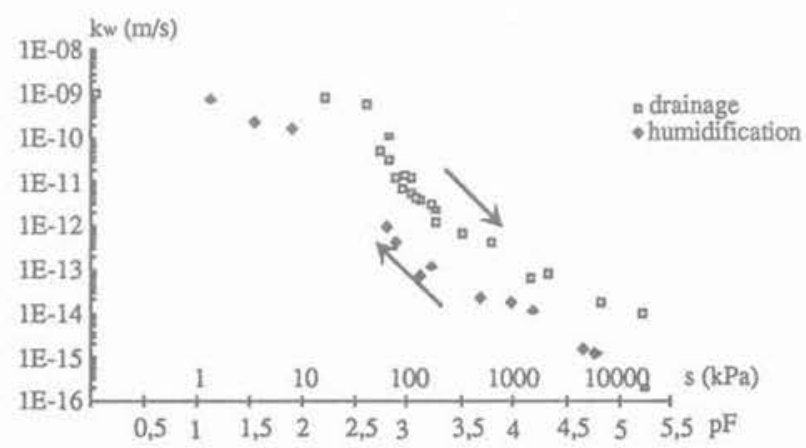

Fig. 5. - Evolution de la conductivité hydraulique au cours d'un cycle drainage-mouillage.

Fig. 5. - Evolution of hydraulic conductivity during a drying-wetting cycle.

\section{MÉTHODE DE LA SURPRESSION D'AIR}

\subsection{Principe}

Une pierre poreuse, perméable à l'eau et imperméable à l'air, sur laquelle repose l'échantillon du sol est placée dans une cellule à pression d'air, sous cette pierre l'eau est maintenue à la pression atmosphérique. La succion produite dans l'échantillon quand le drainage (ou l'humidification) est achevé est égale à la pression d'air exercée sur l'échantillon :

$$
\mathrm{s}=\mathrm{u}_{\mathrm{w}}-\mathrm{u}_{\mathrm{s}}
$$

avec:

$\mathrm{u}_{\mathrm{w}}$, pression d'eau;

$\mathrm{u}_{\mathrm{a}}$, pression d'air;

s, la succion.

\subsection{Dispositif expérimental}

L'étude du comportement des sols partiellement saturés nécessite un appareillage et une technique différents de ceux nécessaires pour l'étude des sols saturés. Ainsi, des techniques ont été élaborées depuis les années soixante par BISHOP (1960), BISHOP et DONALD (1961) qui ont réalisé la première cellule triaxiale à succion im. posée; par la suite, apparaissent d'autres techniques 
d'imposition de la succion telle que la cellule osmotique (DELAGE et al., 1987). Le but visé dans ce travail né. cessite un appareillage qui permet d'imposer une succion et d'enregistrer, avec le maximum de précision, les modifications du volume total de l'échantillon et du volume d'eau induites par l'application de cette succion. Pour ceci on a utilisé un système triaxial équipé de contrôleurs pression-volume type GDS (Geotechnical Digital System) (MENZIES, 1988) (fig. 6), ce dernier comprend :

- une cellule triaxiale: elle est de type hydraulique qui correspond au modèle développé par BISHOP et WESLEY (1975). Cette cellule est équipée d'une pierre céramique dont la valeur d'entrée d'air est de 1500 $\mathrm{kPa}$;

- des contrôleurs numériques digitaux: ce sont des vérins hydrauliques contrôlés par un micro-processeur pour la régulation et la mesure précise de la pression dans le liquide et des variations de volume du liquide. Ils possèdent une capacité de $200 \mathrm{~cm}^{3}$ et permettent d'atteindre une pression de $2000 \mathrm{kPa}$. La mesure de la pression se fait avec une précision de $0,2 \mathrm{kPa}$ et celle du volume avec une précision de $1 \mathrm{~mm}^{3}$; par un sys. tème d'interface, ils sont reliés à un ordinateur qui, d'une façon continue et avec une fréquence qui dépend de la durée d'essai, procéde à l'acquisition et au traitement des données.

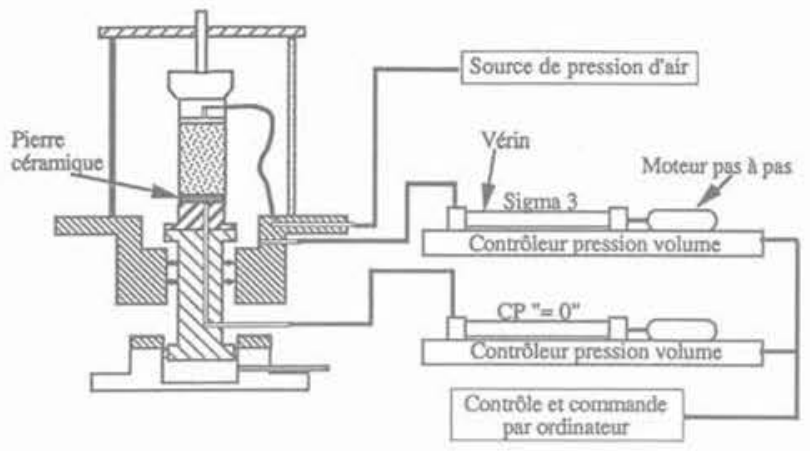

Fig. 6. - Schéma du système triaxial GDS.

Fig. 6. - GDS triaxial testing system.

\subsection{Description des essais}

Un échantillon (diamètre $38 \mathrm{~mm}$; hauteur $55 \mathrm{~mm}$ ) est carotté dans un moule saturé et consolidé statiquement sous une contrainte de $100 \mathrm{kPa}$, il est consolidé ensuite dans la cellule sous une contrainte isotrope de $800 \mathrm{kPa}$. Puis il est désaturé par étape, en augmentant la pression d'air. On peut effectuer un changement de succion lorsque l'équilibre hydrique est atteint. Tous les essais ont été réalisés dans une salle climatisée (température constante et égale à $20^{\circ} \pm 1^{\circ}$ ).

\subsection{Résultats et discussions}

Le tableau 2 regroupe les caractéristiques d'état et hydriques à l'équilibre.

\subsubsection{Aspect hydraulique}

L'équation d'écoulement décrivant le mouvement de l'eau sortant d'un échantillon durant le drainage a été résolue pour un cas simple par GARDNER (1956). On suppose que durant le processus de sortie de l'eau, la conductivité capillaire est approximativement constante et la relation entre la teneur en eau volumique et la pression qui cause la sortie de l'eau est linéaire. Ces deux hypothèses peuvent être admises si l'incrément de pression qui cause la sortie de l'eau est suffisamment petit. En mesurant le volume d'eau qui sort de l'échantillon en fonction du temps et en utilisant la solution de l'équation d'écoulement, la conductivité capillaire peut être calculée pour chaque intervalle de succion.

On signale que la méthode de Gardner ne tient pas compte de la résistance hydraulique de la pierre poreuse; dans notre cas, la pierre a une perméabilité de l'ordre de $10^{-7} \mathrm{~m} / \mathrm{s}$ qui est nettement supérieure à celle de l'échantillon, ceci nous permet donc d'utiliser cette méthode.

La solution complète de l'équation d'écoulement (Richards) a la forme suivante :

$$
\log \left(Q_{0}-Q(t)\right)=\log \left(\frac{8 Q_{0}}{\pi^{2}}\right)-\alpha^{2} D_{w} t
$$

Tableau 2. - Paramètres hydro-mécaniques à la fin de chaque étape de succion. Hydro-mechanical parameters at the end of each suction step.

\begin{tabular}{|c|c|c|c|c|c|c|}
\hline & $\begin{array}{l}\text { Volume } \\
\left(\mathrm{cm}^{3}\right)\end{array}$ & $\begin{array}{l}\text { indice } \\
\text { des vides }\end{array}$ & $\begin{array}{c}\text { Teneur } \\
\text { en eau (\%) }\end{array}$ & $\Delta V \underset{\left(\mathrm{cm}^{3}\right)}{\Delta}$ & $\begin{array}{l}\Delta V \text { eau } \\
\left(\mathrm{cm}^{3}\right)\end{array}$ & $\begin{array}{l}\text { Temps } \\
\text { (heure) }\end{array}$ \\
\hline Etat initial & 62,38 & 0,746 & 28,5 & & & \\
\hline Consolidation à $800 \mathrm{kPa}$ & 47,35 & 0,325 & 13,72 & 15,029 & 14,118 & 69 \\
\hline $\mathrm{S}=100 \mathrm{kPa}$ & 45,10 & 0,262 & 7,61 & 2,243 & 5,836 & 239 \\
\hline $\mathrm{S}=200 \mathrm{kPa}$ & 44,33 & 0,241 & 7,09 & 0,773 & 0,497 & 230 \\
\hline $\mathrm{S}=400 \mathrm{kPa}$ & 43,73 & 0,224 & 6,23 & 0,601 & 0,827 & 240 \\
\hline $\mathrm{S}=600 \mathrm{kPa}$ & 42,62 & 0,204 & 2,77 & 0,706 & 3,301 & 278 \\
\hline
\end{tabular}


$\alpha=(n \pi / 2 L)$ avec $n=1,3,5,7, \ldots$ et $L$ est la longueur de l'échantillon,

$Q_{0}$, flux total sortant durant le processus de l'écoulement,

$\mathrm{Q}(\mathrm{t})$, flux sortant à l'instant $\mathrm{t}$,

$\mathrm{D}_{w}$, la diffusivité.

Cette équation peut être utilisée pour déterminer la diffusivité $\mathrm{D}_{w}$ à partir des données expérimentales, en effet si les valeurs déterminées expérimentalement de $\left(Q_{0}-\right.$ Q) sont tracées en fonction du temps avec une échelle semi-logarithmique, une ligne droite avec une pente égale à $\alpha^{2} D_{u}$ et qui coupe l'axe en $8 Q_{0} / \pi^{2}$ doit être obtenue. $\mathrm{Si}$ la pente de la ligne est notee $\mathrm{B}$, alors $\mathrm{D}_{w}$ $=\mathrm{B} / \alpha^{2}$ et $\mathrm{k}_{w}$ est donnée par l'expression suivante:

$$
\mathrm{k}_{w}=\frac{\mathrm{B} \mathrm{Q}_{0} \rho \mathrm{g}}{\mathrm{V} \Delta \mathrm{P} \alpha^{2}}
$$

$\rho$, masse volumique de l'eau,

$\mathrm{g}$, accélération de la pesanteur,

$\mathrm{V}$, volume de l'échantillon,

$\Delta \mathrm{P}$, incrément de pression.

La figure 7 présente l'évolution de $\log \left(Q_{0}-Q(t)\right)$ en fonction du temps pour deux étapes de désaturation (de 0 à 100 et de 100 à $200 \mathrm{kPa}$ ), les équations de ces deux droites sont obtenues par régression linéaire. Connaissant la pente de ces droites on peut donc calculer la diffusivité et en déduire la conductivité, les valeurs calculées pour les quatre paliers sont:

$\mathrm{S}=100 \mathrm{kPa} \mathrm{D}_{w \mathrm{w}}=8,510^{-11} \mathrm{~m}^{2} / \mathrm{s} \quad \mathrm{k}_{w}=310^{-11} \mathrm{~m} / \mathrm{s}$ $\mathrm{S}=200 \mathrm{kPa} \quad D_{w}^{w}=510^{-11} \mathrm{~m}^{2} / \mathrm{s} \quad \mathrm{k}_{w}^{w}=610^{-12} \mathrm{~m} / \mathrm{s}$ $\mathrm{S}=400 \mathrm{kPa} \quad \mathrm{D}_{\mathrm{w}}^{\mathrm{w}}=3,510^{-11} \mathrm{~m}^{2} / \mathrm{s} \quad \mathrm{k}_{\mathrm{w}}=310^{-12} \mathrm{~m} / \mathrm{s}$ $\mathrm{S}=600 \mathrm{kPa} D_{w}^{\mathrm{w}}=210^{-11} \mathrm{~m}^{2} / \mathrm{s} \quad \mathrm{k}_{\mathrm{w}}=110^{-12} \mathrm{~m} / \mathrm{s}$

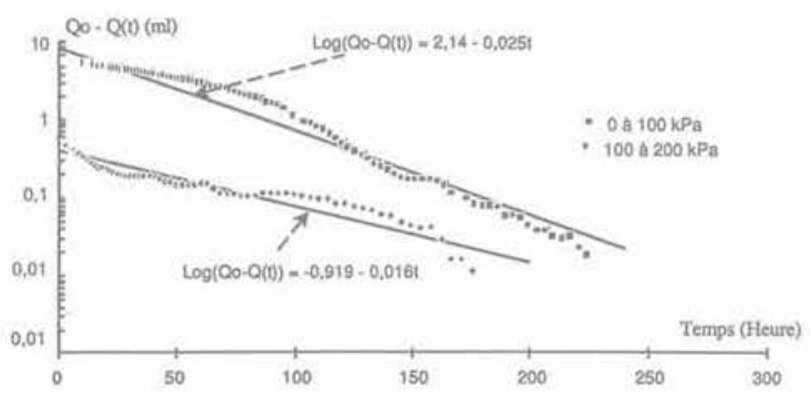

Fig. 7. - Graphe semi-logarithmique du flux sortant en fonction du temps.

Fig. 7. - Outfiow versus time.

La figure 8 montre l'évolution du degré de saturation pour les quatre étapes de désaturation, on remarque une diminution importante du degré de saturation en passant d'une succion de 0 à $100 \mathrm{kPa}$ et de 400 à $600 \mathrm{kPa}$, l'hypothèse d'une valeur constante de la diffusivité est donc non réaliste pour ces deux intervalles de succion.

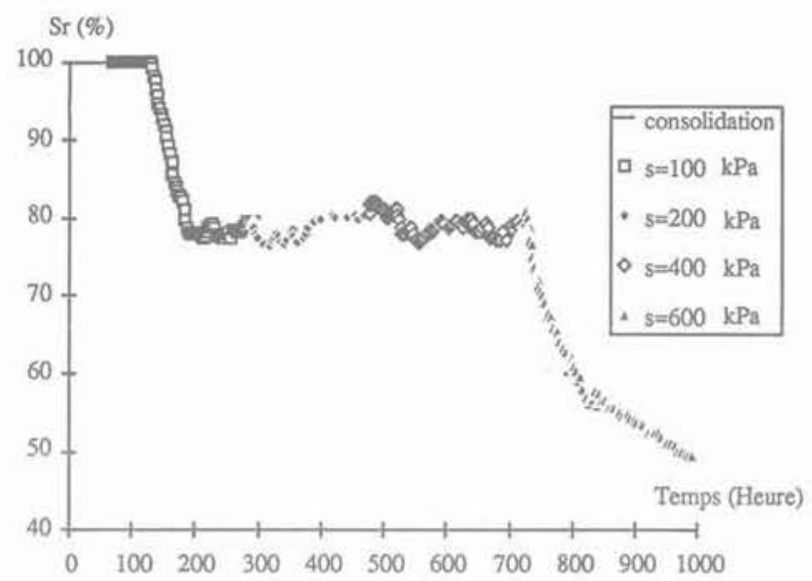

Fig. 8. - Evolution du degré de saturation $\mathrm{Sr}$ au cours du temps pour les 4 étapes de désaturation.

Fig. 8. - Variations of the saturation degree versus time.

\subsubsection{Aspect mécanique}

La déformation d'un sol non saturé est fonction des variables d'état de contrainte (la charge extérieure et la succion) ainsi que du chemin suivi (BARDEN et al., 1969). Un accroissement de succion contribue à la rigidification du sol vis-à-vis de la variation des contraintes appliquées, cette augmentation de la succion peut être interprétée comme un accroissement de pression de préconsolidation apparente (AITCHISON et WOOD. BURN, 1969; DUDLEY, 1970).

La dépendance entre la perméabilité et la consolidation d'un sol saturé est actuellement bien établie, la figure 9 présente un exemple qui montre cette dépendance, on note une variation simultanée typique et égale du volume d'eau drainée et du volume de l'échantillon. En revanche, la variation de ces volumes n'est plus égale en passant d'une succion de 0 à $100 \mathrm{kPa}$ et de 100 à $200 \mathrm{kPa}$ (fig. 10), mais on observe la même allure des courbes. L'augmentation de la succion engendre simultanément une désaturation du sol.

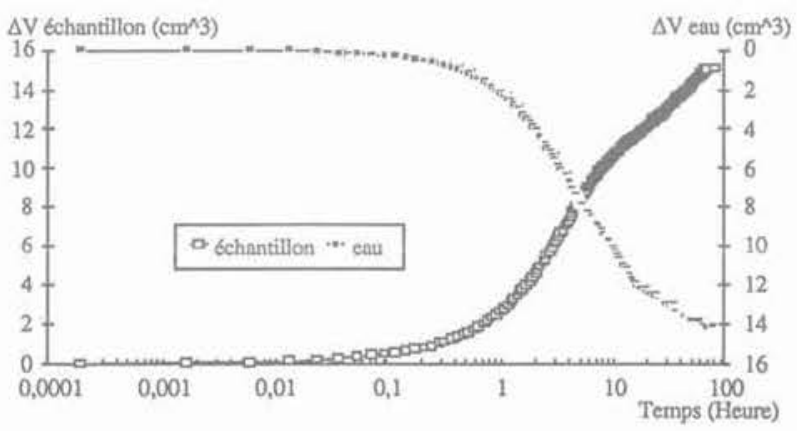

Fig. 9. - Variation volumétrique de l'échantillon et de l'eau lors de la consolidation $\left(\sigma_{3}=800 \mathrm{kPa}\right)$.

Fig. 9. - Volume changes of sample and water during consolidation $\left(\sigma_{3}=800 \mathrm{kPa}\right)$.

On remarque que les équilibres hydrique et mécanique ont lieu simultanément. On peut noter que le système "GDS " permet d'étudier avec une grande précision les phénomènes qui se produisent lors d'une application 


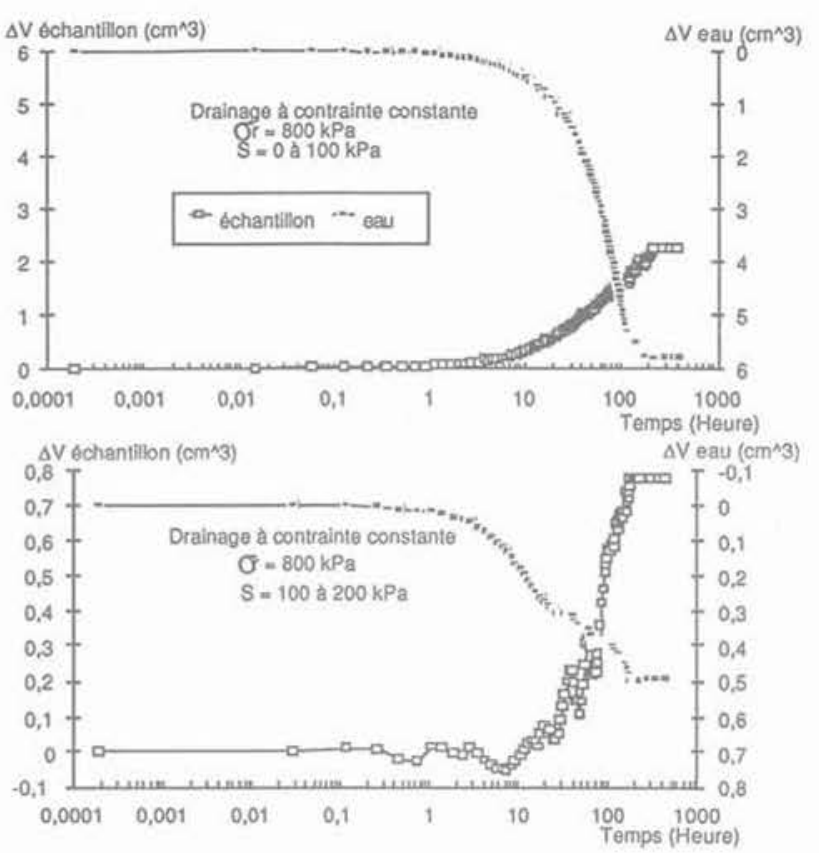

Fig. 10. - Variation du volume de l'échantillon et de la quantité d'eau drainée pour 2 étapes de désaturation.

Fig. 10. - Volume changes of sample and volume of drained water versus time.

de contrainte mécanique ou hydrique et cela malgré les faibles quantités d'eau échangées; on note aussi que l'effet de la succion sur les changements volumiques de l'échantillon est similaire à celui d'une contrainte mé. canique (fig. 11).

Il est intéressant d'observer que les deux enregistrements ( $\Delta V$ eau et $\Delta V$ échantillon) sont bien couplés, en effet, le pourcentage de déformation et le changement du volume d'eau dépendent de la conductivité hydraulique du sol.

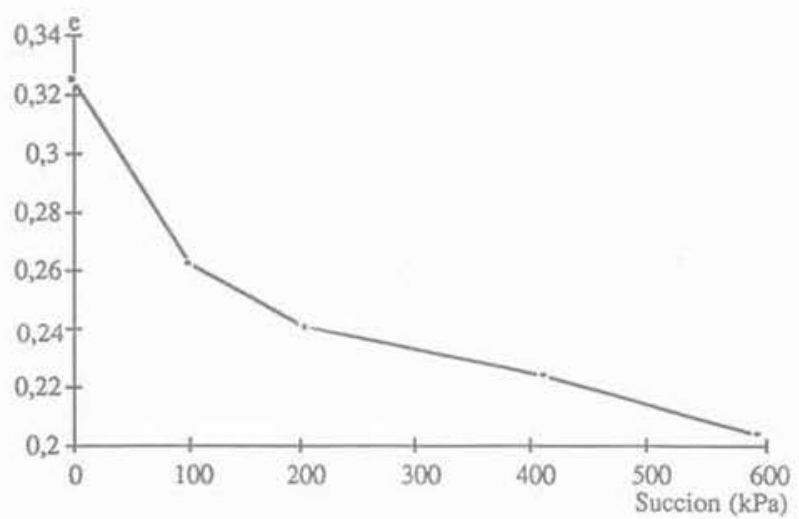

Fig. 11. - Effet de la succion sur l'indice des vides.

Fig. 11 , - Void ratio versus suction.

\section{CONCLUSIONS}

A partir de ces résultats des éléments de réponse pour la compréhension du comportement hydraulique des sols non saturés peuvent être avancés:

- Sur une large gamme de succion pouvant atteindre

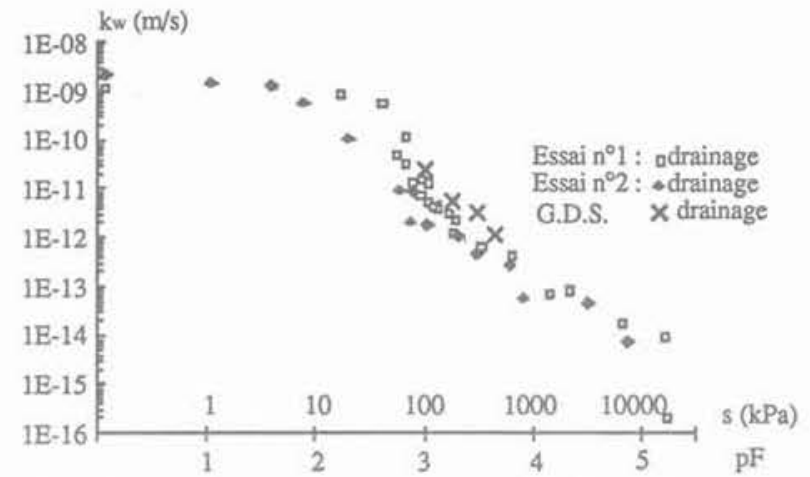

Fig. 12. - Comparaison des conductivités hydrauliques obtenues par les deux méthodes.

Fig. 12, - Comparison of the hydraulic conductivities obtained by both methods.

un $\mathrm{pF}$ de 3 , la conductivité reste égale à celle mesurée à l'état saturé car la diminution de la teneur en eau au début est compensée par la diminution de l'indice des vides;

- Pour un même type de sol et pour des fortes valeurs de succion, la conductivité hydraulique d'un sol dense est proche de celle d'un sol lâche;

- En condition non saturée la conductivité est davan. tage influencée par la teneur en eau et le degré de saturation que par l'indice des vides;

- La succion a un double effet : variations volumiques de l'eau et de l'échantillon qui sont simultanées et non égales;

- Les valeurs de la conductivité hydraulique obtenues par la méthode à surpression d'air sont approximativement les mêrnes que celles obtenues par la méthode des colonnes (fig. 12), cependant les valeurs correspondantes aux deux intervalles 0 à 100 et 400 à $600 \mathrm{kPa}$ sont à prendre en compte avec précaution car le degré de saturation varie considérablement à l'intérieur de ces intervalles;

- On note qu'un des avantages de la méthode des profils instantanés est la simulation des processus des mouvements de l'eau dans les sols, proche de ceux observés dans la nature, auxquels la connaissance de la conductivité hydraulique mesurée peut être logiquement appliquée.

En revanche, la méthode à surpression d'air, telle qu'elle a été mise en cuvre ici, présente des avantages relatifs à une bonne définition des conditions aux limites, à la possibilité de faire varier les états initiaux et à la grande précision des mesures effectuées en continu.

\section{BIBLIOGRAPHIE}

AITCHISON G.D. et WOODBURN J.A. (1969), Soil suction in foundation design. Proc. 7th ICSMFE vol. 2, Mexico, pp. 31-44.

BARDEN L., MUDEDOR A.O. et SIDES G.R. (1969), Volume change characteristics of unsaturated clays. Journal Soil Mech. Found. Div. ASCE. 95 SM1, pp. 33-51. 
BISHOP A.W. (1960), The measurement of pore pressure in the triaxial test. Pore pressure and suction in soil. Butterworths, London, pp. 47-52.

BISHOP A.W. et DONALD I.B. (1961), The experimental study of partly saturated soil in the triaxial apparatus. Proc. 5th ICSMFE vol. 1, Paris, pp. 1321.

BISHOP A.W. et WESLEY (1975), A hydraulique triaxial apparatus for controlled stress path testing. Géotechnique, vol. 25, n4, pp. 657-670.

DANIEL D.E. (1983), Permeability test for unsaturated soil. Geotechnical Testing Journal, ETJODJ, vol. 6, $\mathrm{n}^{\circ} 2$, pp. 81-86.

DELAGE P. SURAJ DE SILVA G.P.R. et DE LAURE E. (1987), A new triaxial apparatus for non saturated soils, $9^{\circ}$ Congrès Européen de Mécanique des Sols, vol. 1, Dublin, pp. 26-28.

DUDLEY J.H. (1970), Review of collapsing soils. Journal Soil Mech. Found. Div., ASCE, 96 (SM3), pp. 925-947.

FRY J.-J. (1992), Les calculs d'écoulement transitoire dans les sols. A la recherche d'une méthodologie. Bilan et perspectives GRECO * Géomatériaux », pp. 95-130.

GARDNER R. (1956), Calculation of capillary conductivity from pressure plate outflow data. Proceedings of the Soil Science Society of America, 20, pp. 317-320.

KLUTE A. (1965), Laboratory measurement of hydraulic conductivity of unsaturated soil. « Method of soil analysis ", Amer. Soc. Agron. Monograph 9, pp. 253-261.
KUNZE R.J. et KIRKHAM D. (1962), Simplified accouting for membrane impedance in capillary conductivity determinations. Soil Sci. Soc. Amer. Proc. 26, pp. 421.426.

MENZIES B.K. (1988), A computer controlled hydraulic triaxial testing system. Autorized Reprint 1988 from special technical publication, pp. 977-988.

MILLER E.E. et EI.RICK D.E. (1958), Dynamic determination of capillary conductivity extended for non-negligible membrane impedance. Soil Sci. Soc. Amer. Proc. 22, pp. 483-486.

PECK A.J. (1966), Diffusivity determination by a new outflow method. IASH/AIHS-Unesco, publication $\mathrm{n}^{\circ} 82$ AIHS, pp. 191-202.

SIBLEY J.W. et WILLIAMS D.J. (1990), A new filter material for measuring soil suction. Geotechnical Testing Journal, GTJODJ, vol. $13, \mathrm{n}^{\circ} 4$, pp. 381 384.

VACHAUD G. (1968), Contribution à l'étude des problèmes d'écoulement en milieux poreux non saturés. Thèse de doctorat, Faculté des Sciences de l'Université de Grenoble, 159 p.

VAUCLIN M. (1975), Etude expérimentale et numérique du drainage de nappes à surface libre. Influence de la zone non saturée. Thèse de doctorat, Université scientifique et médicale de Grenoble, $196 \mathrm{p}$.

WESSELING J. et WIT K.E. (1966), An infiltration method for determination of the capillary conductivity of undisturbed soil cores. IASH/AIHS-Unesco, publication $n^{\circ} 82$ AIHS, pp. 223-234. 